\title{
A 2-D Second-Order Recursive Inverse Adaptive Filtering Algorithm
}

\author{
Mohammad Shukri Salman, Aykut Hocanin, and Osman Kukrer
}

\begin{abstract}
In this paper, a two-dimensional (2-D) version of the recently proposed second order Recursive Inverse (2nd order RI) algorithm is introduced. In the proposed algorithm, instead of estimating the inverse autocorrelation matrix, a second order estimate of the autocorrelation matrix and cross correlation vector, which provide an improved and a more stable performance, are used. Also, the filter coefficients are updated along both the horizontal and vertical directions on a 2-D plane. The performance of the proposed algorithm is compared to that of the 2-D RLS algorithm in Adaptive Line Enhancer (ALE) problem. Simulation results show that the proposed algorithm leads to an improved performance compared to that of the 2-D RLS algorithm.
\end{abstract}

Index Terms-2-D RLS, adaptive filters, ALE.

\section{INTRODUCTION}

Due to increased use of digital imaging and video in consumer electronics and multimedia applications, the LMS adaptive algorithm was extended from 1-D to 2-D and has taken many shapes of implementation. However, these ways update the filter coefficients only along the horizontal direction on a 2-D plane [1]. Consequently, these algorithms cannot sufficiently exploit information in 2-D signals.

Different types of 2-D adaptive algorithms, which can update the filter coefficients both along the horizontal and the vertical directions on a 2-D plane, were later developed and applied to reduce noise in image signals. One of the most efficient algorithms that have shown high performance in image noise removal is the 2-D RLS algorithm [2], [3]. This algorithm suffers from its high computational complexity. Also, it suffers from stability problems when the forgetting factor is small and tracking capability when it is high [4].

In this paper, we propose a new 2-D second order recursive inverse adaptive filtering technique, based on the recently proposed second order recursive inverse algorithm [5], that uses the second order recursive estimate of the autocorrelation matrix and cross-correlation vector which in turn provide an improved performance over that of the 2-D RLS algorithm, and a more stable performance since it does not require the inversion of the autocorrelation matrix.

Manuscript received June 3, 2013; revised August 10, 2013.

M. S. Salman is with the department of Electrical \& Electronic Engineering, Mevlana (Rumi) University, Selcuklu, Konya, Turkey (e-mail: mssalman@mevlana.edu.tr).

A. Hocanin and O. Kukrer are with the department of Electrical \& Electronic Engineering, Eastern Mediterranean University, Gazimagusa, TRNC, via Mersin 10, Turkey (e-mail: aykut.hocanin@emu.edu.tr, osman.kukrer@emu.edu.tr).

\section{REVIEW OF THE SECOND-ORDER RECURSIVE INVERSE ALGORITHM}

In the recently proposed Second-Order Recursive Inverse (RI) adaptive algorithm [5], the weights update equation is given as:

$$
w_{k}=\left[I-\mu_{k} R_{k}\right] w_{k-1}+\mu_{k} \boldsymbol{p}_{k}
$$

with,

$$
\mu_{k}=\frac{\mu_{0}}{\gamma_{k}}, \text { where } \mu_{0}<\mu_{\max } \text { and } \mu_{\max }=\frac{2}{\lambda_{\max }\left(R_{x x}\right)}
$$

where $R_{x x}=E\left\{x_{k} x_{k}^{T}\right\}$, and $\gamma_{k}$ given by

$$
\gamma_{k}=\frac{1}{\beta-1}+\alpha_{1} z_{1}^{k}+\alpha_{2} z_{2}^{k}
$$

where $\alpha_{1}=\frac{\beta-z_{2}}{(1-\beta)\left(z_{2}-z_{1}\right)}, \alpha_{2}=\frac{\beta-z_{1}}{(1-\beta)\left(z_{2}-z_{1}\right)}$. in (1), $\boldsymbol{R}_{k}$ and $\boldsymbol{p}_{k}$ are the instantaneous autocorrelation matrix and cross-correlation vector, respectively. They are estimated recursively, using the second order estimates, as:

$$
\begin{aligned}
& \boldsymbol{R}_{k}=\beta_{1} \boldsymbol{R}_{k-1}+\beta_{2} \boldsymbol{R}_{k-2}+\boldsymbol{x}_{k} \boldsymbol{x}_{k}^{T}, \\
& \boldsymbol{p}_{k}=\beta_{1} \boldsymbol{p}_{k-1}+\beta_{2} \boldsymbol{p}_{k-2}+d_{k} \boldsymbol{x}_{k} .
\end{aligned}
$$

where $\beta_{1}$ and $\beta_{2}$ are the forgetting factors, $\mathbf{x}_{k}$ is the tap-input vector and $d_{k}$ is the desired response.

\section{Proposed Algorithm}

Equation (1) describes the filter weights update equation of the Second-Order RI algorithm [5]. It can be generalized into its 2-D form as:

$$
\boldsymbol{w}_{k}\left(m_{1}, m_{2}\right)=\left[\boldsymbol{I}-\mu_{k} \boldsymbol{R}_{k}\right] \boldsymbol{w}_{k-1}\left(m_{1}, m_{2}\right)+\mu_{k} \boldsymbol{p}_{k},
$$

where $\boldsymbol{w}_{k}\left(m_{1}, m_{2}\right)$ is the 2-D tap-weight vector with dimensions $N \times N, m_{1}=0,1, \ldots, N-1, m_{2}=0,1, \ldots, N-1$, $\boldsymbol{R}_{k}$ and $\boldsymbol{p}_{k}$ are the instantaneous autocorrelation matrix and cross-correlation vector, respectively. They are estimated 
recursively as:

$$
\boldsymbol{R}_{k}=\beta_{1} \boldsymbol{R}_{k-1}+\beta_{2} \boldsymbol{R}_{k-2}+\boldsymbol{x}\left(n_{1}, n_{2}\right) \boldsymbol{x}^{T}\left(n_{1}, n_{2}\right),
$$

and

$$
\boldsymbol{p}_{k}=\beta_{1} \boldsymbol{p}_{k-1}+\beta_{2} \boldsymbol{p}_{k-2}+d\left(n_{1}, n_{2}\right) \boldsymbol{x}\left(n_{1}, n_{2}\right) .
$$

where $d\left(n_{1}, n_{2}\right)$ is the desired output and $\boldsymbol{x}\left(n_{1}, n_{2}\right)$ is the filter input. The filter input $\left(\boldsymbol{x}\left(n_{1}, n_{2}\right)\right)$ and tap-weight vector $\left(\boldsymbol{w}_{k}\left(m_{1}, m_{2}\right)\right)$ can be defined using the following column-ordered vectors [6],

$$
\boldsymbol{x}\left(n_{1}, n_{2}\right)=\left(\begin{array}{c}
x\left(n_{1}, n_{2}\right) \\
\vdots \\
x\left(n_{1}, n_{2}-N+1\right) \\
\vdots \\
x\left(n_{1}-N+1, n_{2}\right) \\
\vdots \\
x\left(n_{1}-N+1, n_{2}-N+1\right)
\end{array}\right)
$$

and,

$$
\boldsymbol{w}_{k}\left(m_{1}, m_{2}\right)=\left(\begin{array}{c}
w_{k}(0,0) \\
\vdots \\
w_{k}(0, N-1) \\
\vdots \\
w_{k}(N-1,0) \\
\vdots \\
w_{k}(N-1, N-1)
\end{array}\right) .
$$

For 2-D applications, there can be a number of ways that data can be reused. One possible way is shown in Fig. 1 [7]. In this scheme, as shown in Fig. 1b, we consider a mask of $3 \times 3$ pixels which move horizontally to the right by one column at a time until the end of each row. Afterward, the same process is repeated with the next row below until the last 9 pixels of the image are reached. At the end of each process of the mask, the data are reshaped as shown in Fig. 1a, starting from the last pixel in the lower right corner.

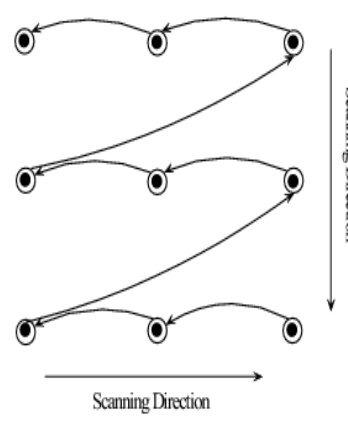

(a)

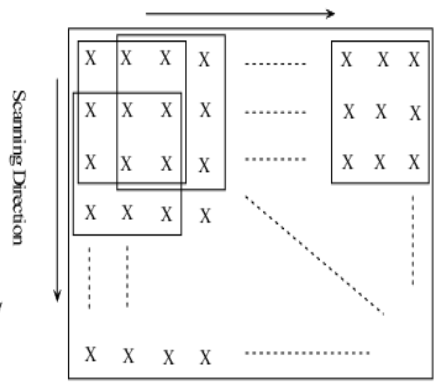

(b)
Fig. 1. Rectangular configuration of data-reusing in 2-D, [7].
The filter output is given by the following 2-D convolution:

$$
y\left(n_{1}, n_{2}\right)=\sum_{m_{1}=0}^{N-1} \sum_{m_{2}=0}^{N-1} w\left(m_{1}, m_{2}\right) x\left(n_{1}-m_{1}, n_{2}-m_{2}\right) .
$$

\section{Simulation Results}

In order to see the performance of the proposed algorithm, it is applied to a 2-D ALE in [7]. In this setting, the original image with noise, $\boldsymbol{x}\left(n_{1}, n_{2}\right)$ is the same as the desired response, $d\left(n_{1}, n_{2}\right)$. The adaptive filter input, $\boldsymbol{u}\left(n_{1}, n_{2}\right)$ is a delayed version of the desired response. The noise added to the original image, $\boldsymbol{I}$ was multiplicative noise using (12),

$$
\boldsymbol{x}=\boldsymbol{I}+v \boldsymbol{I} .
$$

where $v$ is uniformly distributed random noise with zero mean and variance $\sigma_{v}^{2}=5$.

The proposed algorithm is compared to the well-known 2-D RLS algorithm. Both algorithms were implemented using a 2-D FIR filter of size $3 \times 3$ taps. For the proposed algorithm, $\mu_{0}=0.00065, \beta=0.995$ for Fig. $2 \mathrm{c}$ and $\beta=0.999$ for Fig. 2e. For the 2-D RLS algorithm, $\beta=0.995$ for Fig. $2 \mathrm{~d}$ and $\beta=0.999$ for Fig. 2f.

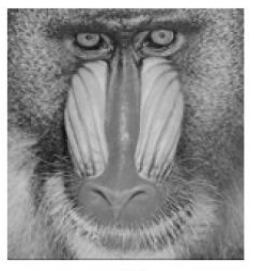

(a)

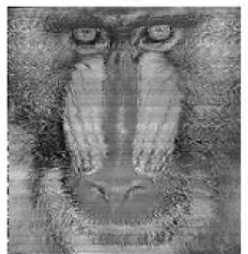

(d)

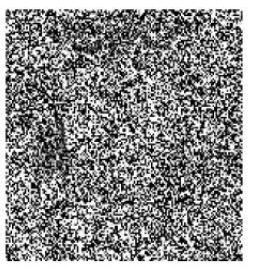

(b)

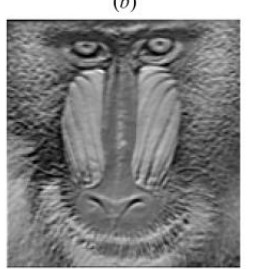

(e)

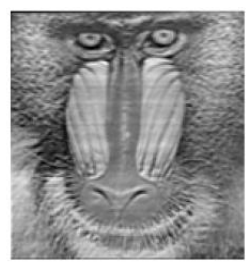

(c)

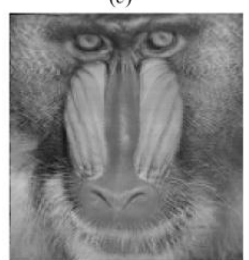

$(f)$
Fig. 2. (a) Original image. (b) Image with noise. (c) Restored image using the proposed algorithm $(\beta=0.995)$. (d) Restored image using the 2-D RLS algorithm $(\beta=0.995)$. (e) Restored image using the the proposed algorithm $(\beta=0.999)$. (f) Restored image using the 2-D RLS algorithm $(\beta=0.999)$.

Fig. 2a shows the original image, Fig. 2b shows the image with noise ('speckle'), Fig. 2c shows the restored image by the proposed 2-D $2^{\text {nd }}$ order RI algorithm with $\beta=0.995$, and Fig. $2 \mathrm{~d}$ shows the restored image by the 2-D RLS algorithm with $\beta=0.995$. We note that the RLS algorithm provides very low performance compared to the proposed algorithm with relatively low values of $\beta$. Fig. 2e shows the restored image by the proposed algorithm with $\beta=0.999$, and Fig. $2 \mathrm{f}$ shows the restored image by the 2-D RLS algorithm with $\beta=0.999$. Even though the value of $\beta$ is very high (close to unity), by inspection, it is clear that the image restored by the proposed algorithm has sharper edges than 
that recovered by the 2-D RLS algorithm.

\section{CONCLUSION}

In this paper, a two-dimensional (2-D) version of the recently proposed second order Recursive Inverse $\left(2^{\text {nd }}\right.$ order $\mathrm{RI}$ ) algorithm is introduced. The proposed approach avoids the use of the inverse autocorrelation matrix in the coefficient update. Instead, a second order estimate of the autocorrelation matrix and cross correlation vector, which provide an improved and a more stable performance, are used. Also, the filter coefficients are updated both along the horizontal and vertical directions on a 2-D plane Simulations show that the proposed algorithm outperforms the 2-D RLS algorithm.

\section{REFERENCES}

[1] M. M. Hadhoud and D. W. Thomas, "The two-dimensional adaptive LMS (TDLMS) algorithm," IEEE Trans. on Circuits and Systems, vol. 35, no. 5, pp. 485-494, 1988.

[2] M. Muneyasu, E. Uemoto, and T. Hinamoto, "A novel 2-D adaptive filter based on the 1-D RLS algorithm," in Proc. IEEE International Symposium on Circuits and Systems, 1997, pp. 2317-2320.

[3] J. Sanubari and K. Tokuda, "RLS-type two-dimensional adaptive filter with a $\mathrm{t}$-distribution assumption," Journal of Signal Processing, vol. 80, no.12, pp. 2483-2495, 2000.

[4] A. H. Sayed, Adaptive Filters, Wiley, New Jersey, 2008.

[5] M. S. Ahmad, O. Kukrer, and A. Hocanin, "Recursive inverse adaptive filter with second order estimation of autocorrelation matrix," in Proc. International Symposium on Signal Processing and Information Technology (ISSPIT), Luxor, Egypt, December 2010, pp. 482-484.

[6] G. O. Glentis, "An efficient affine projection algorithm for 2-D FIR adaptive filtering and linear prediction," Journal of Signal Processing, vol. 86, no. 1, pp. 98-116, January 2006.

[7] M. S. Ahmad, O. Kukrer, and A. Hocanin, "A 2-D recursive inverse adaptive algorithm," Signal Image and Video Processing, Springer, vol. 7, no. 2, pp. 221-226, March 2013.

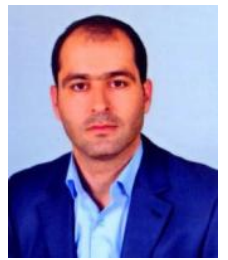

M. S. Salman was born in 1977 in Palestine. He received the B. Sc., M.Sc. and Ph.D. degrees in Electrical and Electronics Engineering from the Eastern Mediterranean University (EMU), in 2006, 2007 and 2011, respectively. From 2006 to 2010, he was a teaching assistant of Electrical and Electronics Engineering department at EMU. In 2010; he joined the Department of Electrical and Electronic Engineering at European University of Lefke (EUL) as a senior lecturer for the Department. Since 2011, he worked as an assist. prof. in the Department of Electrical and Electronic Engineering, Mevlana (Rumi) University, Turkey. He served as a TPC and program chair for many international conferences. He is currently supervising $5 \mathrm{MS}$ and $4 \mathrm{Ph} . \mathrm{D}$. theses. His research interests include signal processing, adaptive filters, image processing, sparse representation of signals, control systems and communications systems.

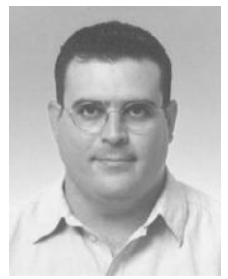

A. Hocanin received the B.S. degree in electrical and computer engineering from Rice University, Houston, TX, USA in 1992 and the M.E. degree from Texas A\&M University, College Station, TX, USA in 1993. $\mathrm{He}$ received the $\mathrm{Ph} . \mathrm{D}$. degree in electrical and electronics engineering from Bogazici University, Istanbul, Turkey in 2000. He received the Cyprus America Scholarship Program (CASP) scholarship from AMIDEAST for his undergraduate education.

He also received the Fahir Ilkel Ph.D. scholarship from Bogazici University. He was a teaching assistant at Texas A\&M, Koc and Isik Universities. He joined the Electrical and Electronic Engineering Department of the Eastern Mediterranean University, Gazimagusa, North Cyprus, in 2000 as an assistant professor. In 2003, he became the vice chairman of the same department. His current research interests include error control coding, multi-user techniques for CDMA and adaptive filtering. He is a senior member of both the Institute of Electrical and Electronics Engineers (IEEE) and the Association of Computing Machinery (ACM). In 2007 and 2012, Aykut Hocanin was promoted to associate professor, and professor, respectively. Since 2007 he is the chairman in the same department.

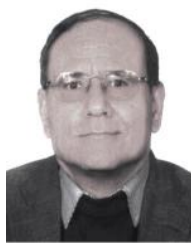

O. Kukrer was born in 1956 in Larnaca, Cyprus. He received the B.S., M.S., and Ph.D. degrees from the Middle East Technical University (METU), Ankara, Turkey, in 1979, 1982, and 1987, respectively, all in electrical engineering. From 1979 to 1985 , he was a research assistant in the Department of Electrical and Electronics Engineering, METU. From 1985 to 1986, he was with the Department of Electrical and Electronics Engineering, Brunel University, London, U.K. He is currently a professor in the Department of Electrical and Electronic Engineering, Eastern Mediterranean University, GaziMagosa, TRNC, Turkey. His research interests include adaptive signal processing, power electronics and control systems. 